\title{
CONTENIDO POLÍNICO DE LA ATMÓSFERA DE ESTEPONA (MÁLAGA): AÑO 1996
}

\author{
F. Javier TORO, M. Mar TRIGO, M. RECIO y B. CABEZUDO
}

\begin{abstract}
RESUMEN. Contenido polínico de la atmósfera de Estepona (Málaga): Año 1996. En el presente trabajo se realiza un estudio del contenido polínico de la atmósfera de Estepona (sur de España) a lo largo del año 1996. El muestreo aerobiológico se ha realizado con la ayuda de un captador tipo Hirst (modelo VPPS-2000 de la casa Lanzoni), colocado a $14,5 \mathrm{~m}$ del nivel del suelo, en el centro de la localidad. Las mayores concentraciones polínicas se registraron durante los meses de Marzo, Abril, Mayo y Junio, periodo en el que se registra casi el 75\% del polen total anual, mientras que los meses de Enero, Septiembre y Diciembre fueron los que detectaron menores cantidades de polen. Los táxones más importantes, en lo que a cantidad de polen se refiere, fueron Cupressaceae, Urtica membranacea y Poaceae, seguidos de Olea, Quercus y Plantago. Los mayores picos de concentración que se produjeron a lo largo del año son debidos principalmente a los aportes de Cupressaceae y Urtica membranacea en Marzo, y Olea y Poaceae en Junio. En todos los meses del año, exceptuando Marzo y Octubre, existe una mayor relación de pólenes procedentes de plantas herbáceas frente a leñosas, así como de plantas silvestres frente a ornamentales.
\end{abstract}

Palabras clave. Aerobiología, polen, Estepona, Sur de España.

ABSTRACT. Pollen analysis content in the atmosphere of Estepona (Málaga): year 1996. In this work, a study about the pollen content in the Estepona (southern Spain) atmosphere was carried out during 1996. The sampling was made with the aid of a volumetric spore trap (model VPPS-2000 by Lanzoni) situated in the city centre at $14,5 \mathrm{~m}$ above ground level. The highest pollen concentrations were detected from March to June, period that registered almost $75 \%$ of total annual pollen; January, September and December being the months with lower pollen counts. The most important taxa, as quantities of pollen regards, were Cupressaceae, Urtica membranacea and Poaceae, followed by Olea, Quercus and Plantago. The highest peaks of pollen concentration were mainly due to the contributions of Cupressaceae and Urtica membranacea in March and Olea and Poaceae, in June. The relationship between woody and herbaceous species pollen as well as between wild and ornamental species pollen is more than one during all months, except March and October when the last concentrations of Cupressaceae pollen are very high.

Key words. Aerobiology, pollen, Estepona, southern Spain.

\section{INTRODUCCIÓN}

Uno de los objetivos de la Red Española de Aerobiología (REA) consiste en realizar un estudio del comportamiento de los aeroalérgenos presentes en el aire de toda la Península Ibérica e Islas Baleares. Por este motivo se comenzó en 1995 un estudio

Este trabajo ha sido financiado por los proyectos PB92-0814-02 de la DGICYT y AMB97-0457-C0705, del Plan Nacional I+D de la CICYT. 
cuantitativo y cualitativo del contenido polínico de la atmósfera de Estepona, localidad de la Costa del Sol situada a $90 \mathrm{~km}$ al oeste de la ciudad de Málaga. Este punto de muestreo pasa a ser, entonces, el más meridional de Europa y más cercano al norte de África. Por otro lado, viene a llenar el vacío existente entre las estaciones costeras de Huelva y Málaga, y amplía el estudio de la atmósfera de la Costa del Sol, que comenzó en Málaga en 1991 (Cabezudo et al., 1994; Recio et al., 1995; Toro et al., 1996, Recio et al., en prensa).

La localidad de Estepona limita al norte con Sierra Bermeja (principal formación europea de peridotitas), al sur con el Mar Mediterráneo, al este con la franja litoral que conduce hacia Marbella y al oeste con el Campo de Gibraltar. Su clima es de tipo mediterráneo húmedo. La temperatura media anual es de $16,8{ }^{\circ} \mathrm{C}$, siendo Enero el mes más frío y Agosto el más cálido, con $11,3{ }^{\circ} \mathrm{C}$ y $23,4{ }^{\circ} \mathrm{C}$ de media, respectivamente. La precipitación media anual es de $556 \mathrm{~mm}$. Al ser una zona muy próxima al estrecho de Gibraltar, los vientos dominante son los de levante (E) y los de poniente (O).

La vegetación circundante consiste fundamentalmente en masas importantes de bosques mediterráneos, preferentemente alcornocales (Quercus suber) y pinares (Pinus pinaster y Pinus pinea). Es importante también, la presencia de matorrales de sustitución como brezales (Erica sp.) y jarales (Cistus sp.). En los cauces de ríos se desarrollan preferentemente alisedas (Alnus glutinosa) y saucedas (Salix sp.). La flora ornamental se encuentra muy bien representada en el interior y los alrededores de la ciudad, dominando entre las distintas esfecies existentes los plátanos de sombra (Platanus $x$ hispanica), moreras (Morus alba), tipuanas (Tipuana speciosa), cipreses (Cupressus sempervirens y Cupressus arizonica) y jacarandas (Jacaranda mimosifolia). En los alrededores de la ciudad existe un matorral ruderal y arvense.

\section{MATERIAL Y MÉTODOS}

El muestreo se ha llevado a cabo durante el año 1996, utilizando un captador volumétrico tipo Hirst (1952), modelo VPPS-2000, de la casa Lanzoni, colocado sobre el tejado del Colegio Público Ramón García de Estepona, a 14,5 metros de altura sobre el nivel del suelo. Este colegio se encuentra situado en la zona centro-oeste de la localidad, sin edificios próximos que impidan la libre circulación del aire. El aparato fue colocado sobre el muro de la fachada, fijado por medio de una pequeña estructura metálica.

Como material adhesivo se ha utilizado aceite de silicona y como medio de montaje glicerogelatina. Para llevar a cabo el recuento se han realizado cuatro barridos por preparación, utilizándose un objetivo de 40x según la metodología propuesta por Domínguez et al. (1991). Los datos obtenidos se expresan en granos de polen por metro cúbico de aire, g.p. $/ \mathrm{m}^{3}$ (media diaria).

Los datos meteorológicos, obtenidos a partir de la estación de Puerto Banús (Marbella), han sido facilitados por el Centro Meteorológico Territorial de Andalucía Oriental. Dicha estación actualmente es la más cercana al punto de muestreo, desde que la de Estepona dejara de funcionar.

Para el estudio de la evolución de la evolución de la relación entre polen de plantas leñosas y herbáceas y entre el polen de ornamentales y naturales, se ha hecho una valoración global, considerándose como especies ornamentales aquéllas que presentan un uso fundamentalmente jardinero o que suelen colocarse en bordes de carreteras.

\section{RESULTADOS Y DISCUSIÓN}

El año 1996 ha sido excepcional en cuanto a precipitaciones, ya que éstas han superado la 


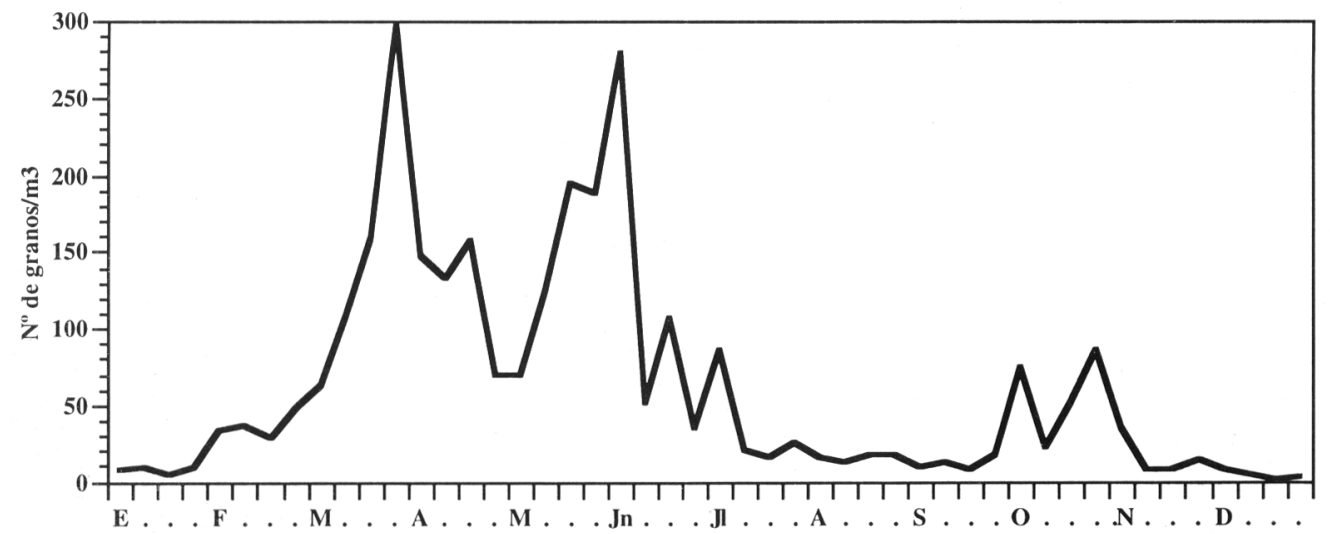

Figura 1. Evolución de la concentración media semanal del polen total registrado en la atmósfera de Estepona durante el año 1996. Total pollen concentration distribution registered in the atmosphere of Estepona during 1996 (weekly average).

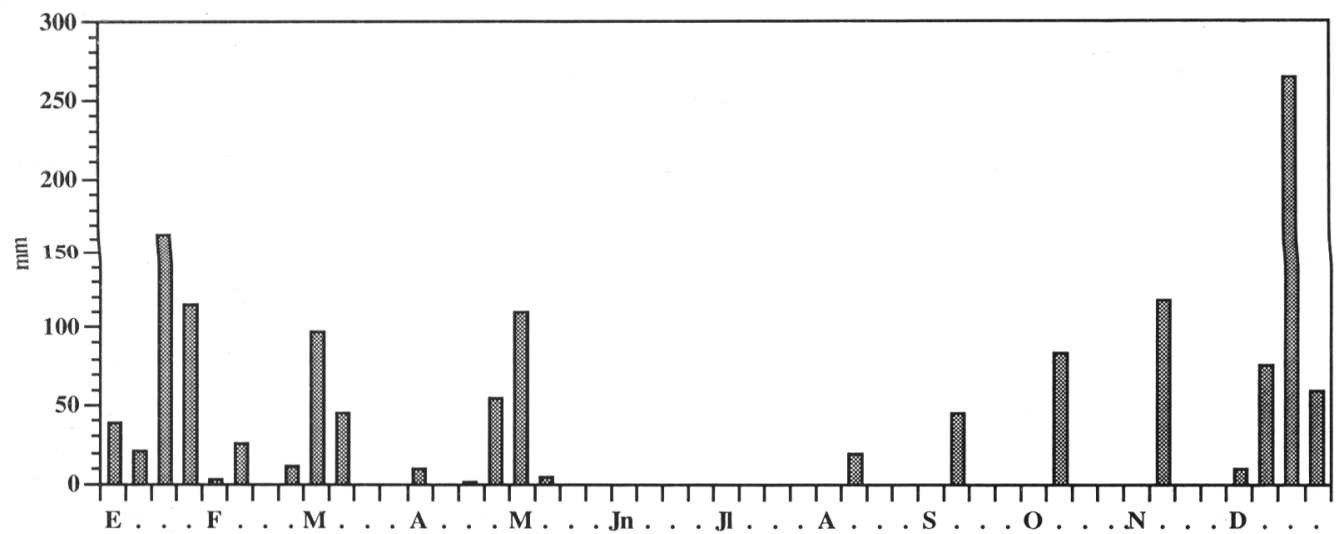

Figura 2. Precipitaciones totales semanales registradas en Puerto Banús (Marbella) durante el año 1996. Weekly total rainfall registered in Puerto Banús (Marbella) during 1996.

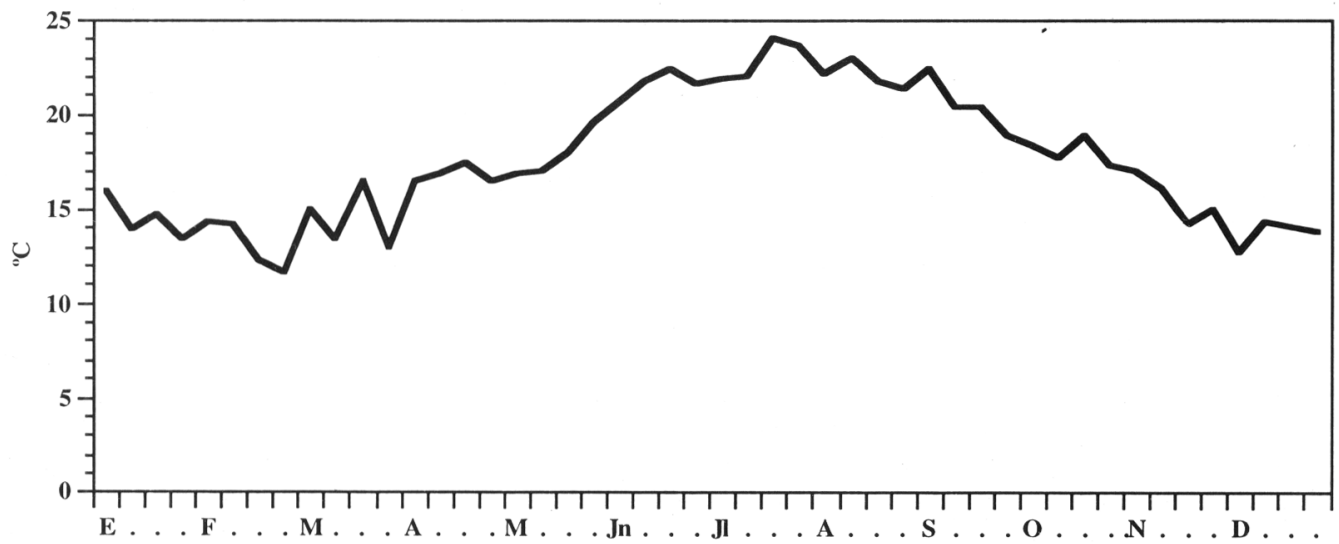

Figura 3. Evolución de la temperatura media semanal registrada en Puerto Banús (Marbella) durante el año 1996. Weekly mean temperature trend registered in Puerto Banús (Marbella) during 1996. 


\begin{tabular}{lrr}
\hline Meses & Total & $\%$ \\
\hline Enero & 254 & 1,13 \\
Febrero & 1082 & 4,81 \\
Marzo & 4957 & 22,03 \\
Abril & 3754 & 16,68 \\
Mayo & 4492 & 19,96 \\
Junio & 3403 & 15,12 \\
Julio & 1183 & 5,26 \\
Agosto & 499 & 2,22 \\
Septiembre & 384 & 1,71 \\
Octubre & 1866 & 8,29 \\
Noviembre & 492 & 2,19 \\
Diciembre & 138 & 0,61 \\
\hline Total anual & 22504 & 100,01 \\
\hline
\end{tabular}

Tabla 1. Valores mensuales absolutos $\left(\mathrm{g} . \mathrm{p} . / \mathrm{m}^{3}\right)$ y relativos (\%), obtenidos por las concentraciones polínicas a lo largo del año 1996. Monthly values (grains $\left./ \mathrm{m}^{3}\right)$ and percentages (\%) obtained by the total pollen counts along 1996.

media de los últimos 15 años (556 mm anuales), alcanzando los $1373 \mathrm{~mm}$. Con respecto a la temperatura, el año estudiado ha sido algo más fresco, siendo Febrero el mes más frío, con $13^{\circ}$ C, y Julio el más cálido con $22,8^{\circ} \mathrm{C}$ (fig. 3).

Durante el año 1996 se han recogido un total de 22504 g.p. $/ \mathrm{m}^{3}$. De éstos se han identificado 35 tipos polínicos que representan el $95,17 \%$ del total de los granos de polen detectados. Los táxones más abundantes en la atmósfera de Estepona durante el año 1996 han sido: Cupressaceae (22,69\%), Urtica membranacea $(15,58 \%)$, Poaceae $(14,10 \%)$, Olea (10,16\%), Quercus (6,03\%), Plantago $(5,42 \%)$, Parietaria $(2,75 \%)$, Pinus $(2,40 \%)$, Chenopodiaceae-Amaranthaceae $(2,21 \%)$ y Rumex $(1,76 \%)$, los cuales en conjunto suman un $83,1 \%$ del polen total anual. Casi las tres cuartas partes del polen total $(73,79 \%)$ se recolectó durante los meses de Marzo, Abril, Mayo y Junio, con un máximo en el mes de Marzo, mientras que el mes de Diciembre fue el que registró menor concentración polínica (tabs. 1, 2 y 3; fig. 1).

Durante los meses de Enero y Febrero se producen las máximas concentraciones de pólenes típicamente invernales como son Alnus y Faxinus, aunque tipos polínicos como Urtica membranacea y Cupressaceae, que ya han comenzando su periodo de polinación, realizan un importante aporte polínico a la atmósfera de Estepona. Durante el mes de Marzo se produce el mayor incremento de polen del año, debido en un $50,39 \%$ al polen de Cupresáceas, que alcanzó su concentración máxima el 29 de Marzo con 494 g.p. $/ \mathrm{m}^{3}$; y en un $34,74 \%$ al polen de Urtica membranacea que alcanzó también su máxima concentración el 27 de Marzo, con 164 g.p. $/ \mathrm{m}^{3}$ (tab. 4). Por otra parte, otros táxones como Platanus, Populus y Ulmus también presentaron sus concentraciones máximas a lo largo de este mes. En el mes de Abril se produjo un importante descenso de las concentraciones totales, a pesar de que algunos táxones alcanzaron sus picos máximos durante este mes, como Betula, Cruciferae, Mercurialis, Morus, Pinus, Pistacia, Quercus, Rumex y Salix, que junto con los aportes de otros táxones como cupresáceas y Urtica membranacea, que están terminando su floración, originaron un pequeño pico de concentración total a mitad del mes de Abril.

A lo largo del mes de Mayo, tras un fuerte descenso de las concentraciones, motivado por la aparición de fuertes precipitaciones (fig. 2), se produce un nuevo incremento de las concentraciones polínicas, debido en un $31,77 \%$ al polen de Olea, que alcanza su día pico el 27 de Mayo con 248 g.p. $/ \mathrm{m}^{3}$; en un $35,78 \%$ al polen de Poaceae y Plantago, que han comenzado su floración; y en un $10,24 \%$ al polen de Quercus, que se mantiene en pleno periodo de floración. En este mes también alcanzan sus picos máximos de concentración otros táxones como Echium y Erica. Durante la primera semana del mes de Junio, las concentraciones de polen aún se mantienen 


\begin{tabular}{|c|c|c|c|c|c|c|c|c|c|c|c|c|c|}
\hline Taxon & Ene & Feb & Mar & Abr & May & Jun & Jul & Ago & Sep & Oct & Nov & Dic & Total \\
\hline Alnus & 6 & 52 & 8 & 0 & 0 & 0 & 0 & 0 & 0 & 1 & 0 & 1 & 68 \\
\hline Apiaceae & 0 & 0 & 0 & 0 & 18 & 78 & 61 & 15 & 6 & 5 & 3 & 0 & 186 \\
\hline Artemisia & 5 & 4 & 4 & 0 & 1 & 0 & 1 & 10 & 14 & 54 & 71 & 21 & 185 \\
\hline Betula & 0 & 0 & 7 & 11 & 3 & 0 & 2 & 1 & 0 & 0 & 0 & 0 & 24 \\
\hline Cannabis & 0 & 0 & 0 & 0 & 5 & 12 & 8 & 2 & 1 & 0 & 0 & 0 & 28 \\
\hline Castanea & 1 & 0 & 1 & 5 & 18 & 81 & 81 & 17 & 5 & 4 & 0 & 0 & 213 \\
\hline Casuarina & 8 & 6 & 0 & 0 & 0 & 0 & 0 & 0 & 8 & 16 & 3 & 1 & 42 \\
\hline Compositae* & 1 & 0 & 3 & 13 & 37 & 95 & 37 & 25 & 18 & 25 & 15 & 3 & 272 \\
\hline Cruciferae & 2 & 5 & 13 & 30 & 18 & 8 & 0 & 4 & 0 & 1 & 3 & 1 & 85 \\
\hline Cupressaceae & 15 & 119 & 2498 & 439 & 29 & 36 & 8 & 6 & 90 & 1563 & 267 & 35 & 5105 \\
\hline Cyperaceae & 0 & 0 & 2 & 1 & 12 & 39 & 19 & 5 & 4 & 0 & 1 & 0 & 83 \\
\hline Chenop.-Amaranth. & 0 & 2 & 6 & 44 & 77 & 77 & 68 & 99 & 76 & 29 & 16 & 4 & 498 \\
\hline Echium & 0 & 0 & 0 & 16 & 118 & 67 & 16 & 2 & 0 & 0 & 0 & 0 & 219 \\
\hline Ericaceae & 1 & 0 & 22 & 28 & 36 & 13 & 0 & 1 & 1 & 2 & 0 & 1 & 105 \\
\hline Fraxinus & 5 & 46 & 2 & 5 & 0 & 0 & 0 & 0 & 0 & 2 & 22 & 16 & 98 \\
\hline Ligustrum & 0 & 0 & 0 & 0 & 0 & 1 & 12 & 3 & 3 & 2 & 0 & 0 & 21 \\
\hline Mercurialis & 5 & 12 & 27 & 41 & 12 & 7 & 0 & 0 & 0 & 0 & 2 & 3 & 109 \\
\hline Morus & 0 & 0 & 26 & 128 & 7 & 0 & 0 & 0 & 0 & 0 & 0 & 0 & 161 \\
\hline Myrtaceae & 1 & 3 & 1 & 10 & 6 & 47 & 147 & 18 & 6 & 7 & 7 & 4 & 257 \\
\hline Olea & 0 & 0 & 7 & 156 & 1427 & 591 & 57 & 26 & 11 & 6 & 3 & 2 & 2286 \\
\hline Palmae & 2 & 14 & 29 & 29 & 8 & 28 & 32 & 37 & 27 & 15 & 14 & 3 & 238 \\
\hline Parietaria & 46 & 64 & 94 & 102 & 71 & 127 & 39 & 13 & 13 & 15 & 18 & 17 & 619 \\
\hline Pinus & 0 & 20 & 87 & 275 & 57 & 79 & 13 & 4 & 3 & 0 & 1 & 0 & 539 \\
\hline Pistacia & 0 & 0 & 10 & 76 & 1 & 0 & 0 & 0 & 0 & 0 & 0 & 0 & 87 \\
\hline Plantago & 0 & 1 & 9 & 236 & 604 & 300 & 46 & 12 & 6 & 3 & 2 & 1 & 1220 \\
\hline Platanus & 0 & 1 & 70 & 34 & 1 & 4 & 1 & 0 & 0 & 0 & 0 & 0 & 111 \\
\hline Poaceae & 3 & 25 & 48 & 214 & 1003 & 1218 & 395 & 143 & 65 & 37 & 13 & 8 & 3172 \\
\hline Populus & 1 & 16 & 35 & 7 & 0 & 0 & 0 & 0 & 0 & 0 & 0 & 0 & 59 \\
\hline Quercus & 18 & 91 & 126 & 486 & 460 & 119 & 13 & 16 & 1 & 3 & 16 & 8 & 1357 \\
\hline Ricinus & 6 & 1 & 0 & 1 & 1 & 4 & 1 & 0 & 0 & 0 & 0 & 0 & 14 \\
\hline Rumex & 1 & 7 & 26 & 152 & 120 & 78 & 3 & 3 & 2 & 2 & 0 & 1 & 395 \\
\hline Salix & 0 & 2 & 2 & 10 & 1 & 0 & 5 & 0 & 0 & 0 & 0 & 1 & 21 \\
\hline Typha & 0 & 0 & 0 & 0 & 0 & 3 & 2 & 0 & 0 & 0 & 0 & 0 & 5 \\
\hline Ulmus & 1 & 4 & 12 & 11 & 0 & 0 & 0 & 0 & 0 & 0 & 0 & 0 & 28 \\
\hline Urtica membranacea & 113 & 557 & 1722 & 974 & 124 & 7 & 0 & 0 & 0 & 1 & 5 & 4 & 3507 \\
\hline Otros & 13 & 30 & 60 & 220 & 217 & 284 & 116 & 37 & 24 & 73 & 10 & 3 & 1087 \\
\hline
\end{tabular}

Tabla 2. Sumas mensuales y anuales de las concentraciones medias diarias de polen $\left(\mathrm{g} . \mathrm{p} . / \mathrm{m}^{3}\right)$ obtenidas por los distintos taxones estudiados durante el año 1996. Monthly and annual sum of the daily average concentrations $\left(\right.$ grains $/ \mathrm{m}^{3}$ ) rise by the different taxa during 1996.

*Excluido Artemisia. Excluding Artemisia.

altas, produciéndose el segundo gran pico anual de concentración media semanal. A partir de ahí las cantidades de polen total comenzaron a descender, aunque se siguen detectando importantes concentraciones diarias de polen de Poaceae (433 g.p. $/ \mathrm{m}^{3}$ ) y Plantago (70 g.p./ $\mathrm{m}^{3}$ ). A mediados de Junio y principios de Julio, aún se producen picos de menor intensidad, originados por los aportes de polen de Poaceae y Parietaria, así como de táxones típicamente veraniegos como Apiaceae, Castanea, Compositae, Cyperaceae, Ligustrum o Myrtaceae. Por otra parte, taxones como Olea, Pinus, Plantago y Quercus todavía alcanzan 


\begin{tabular}{|c|c|c|c|c|c|c|c|c|c|c|c|c|c|}
\hline Taxon & Ene & Feb & Mar & Abr & May & Jun & Jul & Ago & Sep & Oct & Nov & Dic & Total \\
\hline Alnus & 2,36 & 4,81 & 0,16 & 0,00 & 0,00 & 0,00 & 0,00 & 0,00 & 0,00 & 0,05 & 0,00 & 0,72 & 0,30 \\
\hline Apiaceae & 0,00 & 0,00 & 0,00 & 0,00 & 0,40 & 2,29 & 5,16 & 3,01 & 1,56 & 0,27 & 0,61 & 0,00 & 0,83 \\
\hline Artemisia & 1,97 & 0,37 & 0,08 & 0,00 & 0,02 & 0,00 & 0,08 & 2,00 & 3,65 & 2,89 & 14,43 & 15,22 & 0,82 \\
\hline Betula & 0,00 & 0,00 & 0,14 & 0,29 & 0,07 & 0,00 & 0,17 & 0,20 & 0,00 & 0,00 & 0,00 & 0,00 & 0,11 \\
\hline Cannabis & 0,00 & 0,00 & 0,00 & 0,00 & 0,11 & 0,35 & 0,68 & 0,40 & 0,26 & 0,00 & 0,00 & 0,00 & 0,12 \\
\hline Castanea & 0,39 & 0,00 & 0,02 & 0,13 & 0,40 & 2,38 & 6,85 & 3,41 & 1,30 & 0,21 & 0,00 & 0,00 & 0,95 \\
\hline Casuarina & 3,15 & 0,55 & 0,00 & 0,00 & 0,00 & 0,00 & 0,00 & 0,00 & 2,08 & 0,86 & 0,61 & 0,72 & 0,19 \\
\hline Compositae* & 0,39 & 0,00 & 0,06 & 0,35 & 0,82 & 2,79 & 3,13 & 5,01 & 4,69 & 1,34 & 3,05 & 2,17 & 1,21 \\
\hline Cruciferae & 0,79 & 0,46 & 0,26 & 0,80 & 0,40 & 0,24 & 0,00 & 0,80 & 0,00 & 0,05 & 0,61 & 0,72 & 0,38 \\
\hline Cupressaceae & 5,91 & 11,00 & 50,39 & 11,69 & 0,65 & 1,06 & 0,68 & 1,20 & 23,44 & 83,76 & 54,27 & 25,36 & 22,68 \\
\hline Cyperaceae & 0,00 & 0,00 & 0,04 & 0,03 & 0,27 & 1,15 & 1,61 & 1,00 & 1,04 & 0,00 & 0,20 & 0,00 & 0,37 \\
\hline Chenop.-Amaranth. & 0,00 & 0,18 & 0,12 & 1,17 & 1,71 & 2,26 & 5,75 & 19,84 & 19,79 & 1,55 & 3,25 & 2,90 & 2,21 \\
\hline Echium & 0,00 & 0,00 & 0,00 & 0,43 & 2,63 & 1,97 & 1,35 & 0,40 & 0,00 & 0,00 & 0,00 & 0,00 & 0,97 \\
\hline Ericaceae & 0,39 & 0,00 & 0,44 & 0,75 & 0,80 & 0,38 & 0,00 & 0,20 & 0,26 & 0,11 & 0,00 & 0,72 & 0,47 \\
\hline Fraxinus & 1,97 & 4,25 & 0,04 & 0,13 & 0,00 & 0,00 & 0,00 & 0,00 & 0,00 & 0,11 & 4,47 & 11,59 & 0,44 \\
\hline Ligustrum & 0,00 & 0,00 & 0,00 & 0,00 & 0,00 & 0,03 & 1,01 & 0,60 & 0,78 & 0,11 & 0,00 & 0,00 & 0,09 \\
\hline Mercurialis & 1,97 & 1,11 & 0,54 & 1,09 & 0,27 & 0,21 & 0,00 & 0,00 & 0,00 & 0,00 & 0,41 & 2,17 & 0,48 \\
\hline Morus & 0,00 & 0,00 & 0,52 & 3,41 & 0,16 & 0,00 & 0,00 & 0,00 & 0,00 & 0,00 & 0,00 & 0,00 & 0,72 \\
\hline Myrtaceae & 0,39 & 0,28 & 0,02 & 0,27 & 0,13 & 1,38 & 12,43 & 3,61 & 1,56 & 0,38 & 1,42 & 2,90 & 1,14 \\
\hline Olea & 0,00 & 0,00 & 0,14 & 4,16 & 31,77 & 17,37 & 4,82 & 5,21 & 2,86 & 0,32 & 0,61 & 1,45 & 10,16 \\
\hline Palmae & 0,79 & 1,29 & 0,59 & 0,77 & 0,18 & 0,82 & 2,70 & 7,41 & 7,03 & 0,80 & 2,85 & 2,17 & 1,06 \\
\hline Parietaria & 18,11 & 5,91 & 1,90 & 2,72 & 1,58 & 3,73 & 3,30 & 2,61 & 3,39 & 0,80 & 3,66 & 12,32 & 2,75 \\
\hline Pinus & 0,00 & 1,85 & 1,76 & 7,33 & 1,27 & 2,32 & 1,10 & 0,80 & 0,78 & 0,00 & 0,20 & 0,00 & 2,40 \\
\hline Pistacia & 0,00 & 0,00 & 0,20 & 2,02 & 0,02 & 0,00 & 0,00 & 0,00 & 0,00 & 0,00 & 0,00 & 0,00 & 0,39 \\
\hline Plantago & 0,00 & 0,09 & 0,18 & 6,29 & 13,45 & 8,82 & 3,89 & 2,40 & 1,56 & 0,16 & 0,41 & 0,72 & 5,42 \\
\hline Platanus & 0,00 & 0,09 & 1,41 & 0,91 & 0,02 & 0,12 & 0,08 & 0,00 & 0,00 & 0,00 & 0,00 & 0,00 & 0,49 \\
\hline Poaceae & 1,18 & 2,31 & 0,97 & 5,70 & 22,33 & 35,79 & 33,39 & 28,66 & 16,93 & 1,98 & 2,64 & 5,80 & 14,10 \\
\hline Populus & 0,39 & 1,48 & 0,71 & 0,19 & 0,00 & 0,00 & 0,00 & 0,00 & 0,00 & 0,00 & 0,00 & 0,00 & 0,26 \\
\hline Quercus & 7,09 & 8,41 & 2,54 & 12,95 & 10,24 & 3,50 & 1,10 & 3,21 & 0,26 & 0,16 & 3,25 & 5,80 & 6,03 \\
\hline Ricinus & 2,36 & 0,09 & 0,00 & 0,03 & 0,02 & 0,12 & 0,08 & 0,00 & 0,00 & 0,00 & 0,00 & 0,00 & 0,06 \\
\hline Rumex & 0,39 & 0,65 & 0,52 & 4,05 & 2,6 & 2,29 & 0,25 & 0,60 & 0,52 & 0,11 & 0,00 & 0,72 & 1,76 \\
\hline Salix & 0,00 & 0,18 & 0,04 & 0,27 & 0,02 & 0,00 & 0,42 & 0,00 & 0,00 & 0,00 & 0,00 & 0,72 & 0,09 \\
\hline Typha & 0,00 & 0,00 & 0,00 & 0,00 & 0,00 & 0,09 & 0,17 & 0,00 & 0,00 & 0,00 & 0,00 & 0,00 & 0,02 \\
\hline Ulmus & 0,39 & 0,37 & 0,24 & 0,29 & 0,00 & 0,00 & 0,00 & 0,00 & 0,00 & 0,00 & 0,00 & 0,00 & 0,12 \\
\hline Urtica membranacea & 44,49 & 51,48 & 34,74 & 25,95 & 2,76 & 0,21 & 0,00 & 0,00 & 0,00 & 0,05 & 1,02 & 2,90 & 15,58 \\
\hline Suma & 94,88 & 97,23 & 98,79 & 94,14 & 95,17 & 91,65 & 90,19 & 92,59 & 93,75 & 96,09 & 97,97 & 97,83 & 95,17 \\
\hline
\end{tabular}

Tabla 3. Porcentajes mensuales y anuales de los taxones estudiados con respecto al polen total obtenido durante el año 1996. Monthly and annual percentage of the studied taxa in relation with the total pollen obtained during 1996.

*Excluido Artemisia. Excluding Artemisia.

concentraciones apreciables.

Los meses de Agosto y Septiembre fueron meses de muy baja concentración polínica, aunque durante este mes se produjeron las mayores concentraciones mensuales de Chenopodiaceae-Amaranthaceae y Palmae (tab. 2). Durante el mes de Octubre se produce un nuevo pico de concentración total debido fundamentalmente al aporte del polen de Cupresaceae. También en este mes se detectan las máximas concentraciones de Casuarina. A lo largo de los meses de Noviembre y Diciembre decrecen la concentraciones de la mayoría de los táxones, excepto en el caso de Artemisia, que registró durante el mes de Noviembre sus máximas concentraciones.

El comportamiento general que presentan los distintos tipos polínicos en la atmósfera de Estepona es similar al de Málaga (Cabezudo et al., 1994; Recio et al., 1995; Toro et al., 1996), sin embargo existen algunas diferencias entre ambas estaciones, destacando en Estepona la presencia de mayores concentraciones de polen de Urtica membranacea; Alnus, Castanea, Ericaceae y Ulmus, así como menores de Casuarina y Olea. 


\begin{tabular}{lcccc}
\hline Taxón & Día pico & $\begin{array}{c}\text { Concentración } \\
\text { media diaria }\end{array}$ & Semanana pico & $\begin{array}{c}\text { Concentración } \\
\text { media semanal }\end{array}$ \\
\hline Urtica membranacea & 27 Marzo & 164 & $4^{\text {a Marzo }}$ & 110 \\
Cupresaceae & 29 Marzo & 494 & $4^{\text {a Marzo }}$ & 139 \\
Rumex & 7 Abril & 17 & $3^{\text {a Abril }}$ & 7 \\
Pinus & 17 Abril & 69 & $3^{\text {a Abril }}$ & 16 \\
Quercus & 17 Abril & 125 & $3^{\text {a Abril }}$ & 32 \\
Olea & 27 Mayo & 248 & $4^{\text {a Mayo }}$ & 66 \\
Poaceae & 2 Junio & 433 & $1^{\text {a Junio }}$ & 123 \\
Plantago & 2 Junio & 70 & $3^{\text {a Mayo }}$ & 31 \\
Chenop.-Amar. & 2 Junio & 12 & $3^{\text {a Agosto }}$ & 5 \\
Parietaria* & 15 Junio & 13 & $1^{\text {a Junio }}$ & 6 \\
\hline
\end{tabular}

Tabla 4. Valores máximos obtenidos por los 10 taxones más relevantes en la atmósfera de Estepona durante el año 1996. Maximum values obtained by the 10 more important taxa in the atmosphere of Estepona during 1996 (day and week peak).

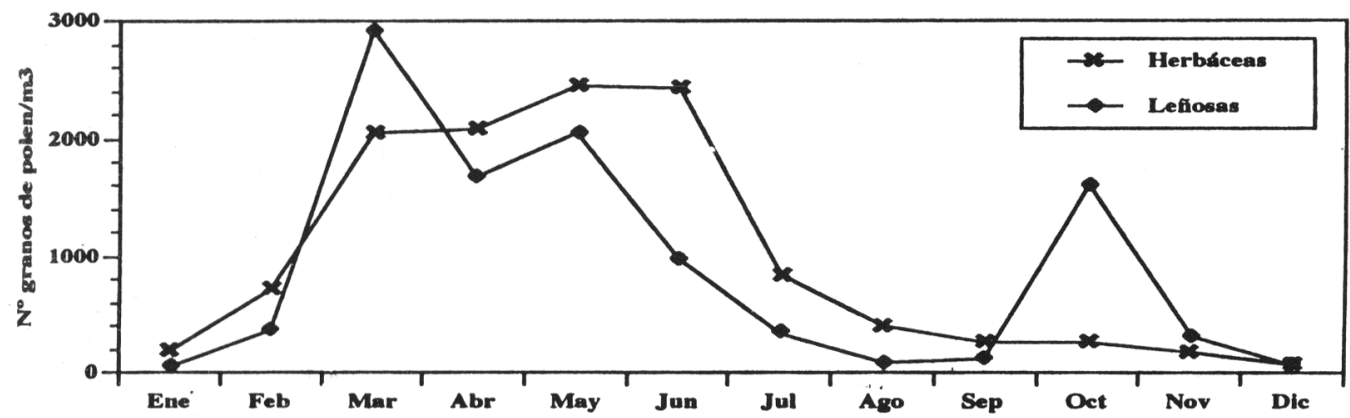

Figura 4. Distribución mensual de las concentraciones obtenidas por el polen de especies leñosas frente al de las herbáceas a lo largo del año 1996. Monthly values trend obtained by the pollen of woody and herbaceous plants during 1996.

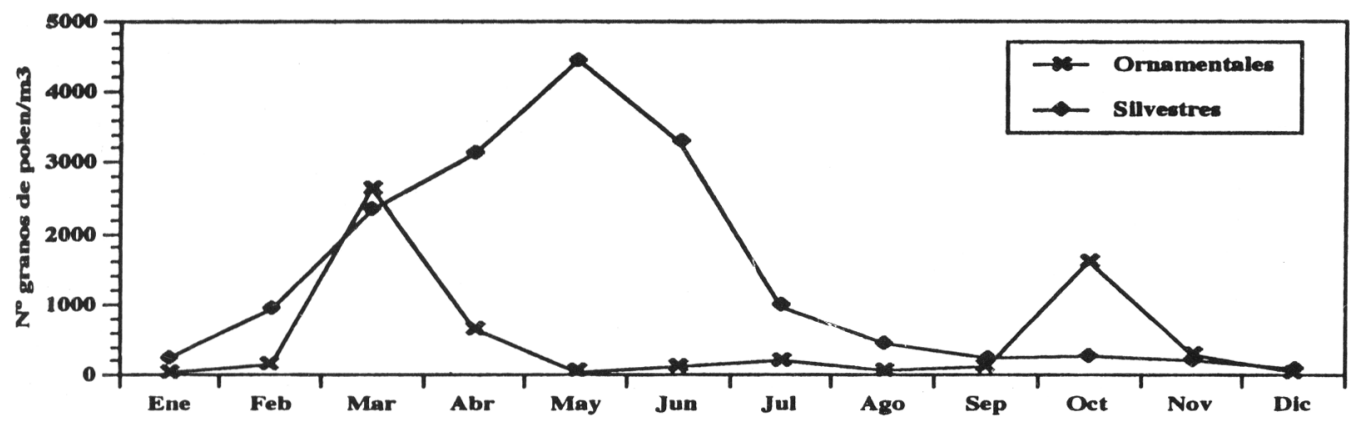

Figura 5. Distribución mensual de las concentraciones obtenidas por el polen de especies ornamentales frente al de silvestres a lo largo del año 1996. Monthly values trend obtained by the pollen of wild and ornamental plants during 1996. 
Se ha detectado un claro predominio de las especies herbáceas frente a las leñosas durante la mayor parte del año (fig. 4), excepto en los meses de Marzo y Octubre, debido, en ambos casos, al fuerte incremento que experimentó el polen de Cupressaceae, que llegó a suponer el 50,39 y el 83,81\% del total mensual, respectivamente (tab. 3). Igualmente, se ha encontrado un fuerte predominio del polen de las especies silvestres frente al de las especies ornamentales (fig. 5), este último presenta dos picos de concentración bien definidos, uno en Marzo y otro en Octubre, debidos, al igual que en el caso anterior, al incremento del polen de Cupresaceae, fundamentalmente.

AGRADECIMIENTOS. Los autores desean agradecer a las Consejerías de Salud y Educación de la Junta de Andalucía la colaboración prestada a la Red Andaluza de Aerobiología (RAA) para el desarrollo de este trabajo.

\section{BIBLIOGRAFÍA}

CABEZUDO, B., M.M. TRIGO, M. RECIO y F.J. TORO -1994- Contenido polínico de la atmósfera de Málaga: Años 1992 y 1993. Acta Bot. Malacitana 19:137-144.

DOMÍNGUEZ VILCHES, E., C. GALÁN SOLDEVILLA, F. VILLAMANDOS DE LA TORRE \& F. INFANTE GARCÍA PANTALEÓN - 1991-Handling and evaluation of the data from the aerobiological sampling. Monografías REA/EAN 1:1-18.
HIRST, J.M. - 1952- An automatic volumetric spore trap. Ann. Appl. Biol. 39 (2): 257-265.

RECIO, M., M.M. TRIGO, F.J. TORO y B. CABEZUDO - 1995- Contenido polínico de la atmósfera de Málaga: A.ño 1994. Acta Bot. Malacitana 20:83-90.

RECIO, M., B. CABEZUDO, M.M. TRIGO y F.J. TORO. Pollen calendar of Málaga (Southern Spain), 1991-1995. Aerobiologia. En prensa.

TORO, F.J., M. RECIO, M.M TRIGO y B. CABEZUDO - 1996- Contenido polínico de la atmósfera de Málaga: Año 1995. Acta Bot. Malacitana 21:57-63.

Aceptado para publicación en Octubre 1997

Dirección de los autores. Dpto. Biología Vegetal. Fac. Ciencias. Universidad de Málaga. Apdo. 59. 29080, Málaga, E-mail: aerox@uma.es 